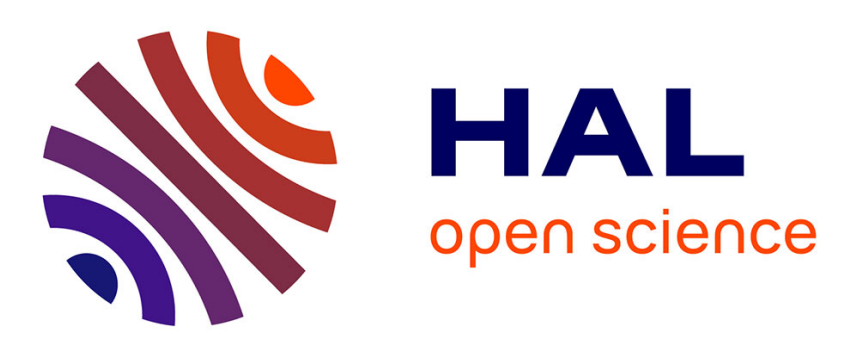

\title{
High bandwidth noninvasive measurements of the linear viscoelasticity of collagen gels
}

\author{
M. Verhulsel, S. Shivokhin, A. Simon, S. Descroix, Christian Frétigny, L.
}

Talini

\section{- To cite this version:}

M. Verhulsel, S. Shivokhin, A. Simon, S. Descroix, Christian Frétigny, et al.. High bandwidth noninvasive measurements of the linear viscoelasticity of collagen gels. Journal of Rheology, 2016, 60 (6), pp.1269 - 1278. 10.1122/1.4965039 . hal-01393439

\section{HAL Id: hal-01393439 \\ https://hal.sorbonne-universite.fr/hal-01393439}

Submitted on 7 Nov 2016

HAL is a multi-disciplinary open access archive for the deposit and dissemination of scientific research documents, whether they are published or not. The documents may come from teaching and research institutions in France or abroad, or from public or private research centers.
L'archive ouverte pluridisciplinaire HAL, est destinée au dépôt et à la diffusion de documents scientifiques de niveau recherche, publiés ou non, émanant des établissements d'enseignement et de recherche français ou étrangers, des laboratoires publics ou privés. 


\section{JOURNAL OF RH=OLOGY}

High bandwidth noninvasive measurements of the linear viscoelasticity of collagen gels

M. Verhulsel, M. E. Shivokhin, A. Simon, S. Descroix, C. Frétigny, and L. Talini

Citation: Journal of Rheology 60, 1269 (2016); doi: 10.1122/1.4965039

View online: http://dx.doi.org/10.1122/1.4965039

View Table of Contents: http://scitation.aip.org/content/sor/journal/jor2/60/6?ver=pdfcov

Published by the The Society of Rheology

\section{Articles you may be interested in}

Viscoelasticity of thermoreversible gelatin gels from mammalian and piscine collagens

J. Rheol. 44, 871 (2000); 10.1122/1.551118

Rheology of reconstituted type I collagen gel in confined compression

J. Rheol. 41, 971 (1997); 10.1122/1.550817

Linear Viscoelasticity at the Gel Point of a Crosslinking PDMS with Imbalanced Stoichiometry

J. Rheol. 31, 683 (1987); 10.1122/1.549955

Analysis of Linear Viscoelasticity of a Crosslinking Polymer at the Gel Point

J. Rheol. 30, 367 (1986); 10.1122/1.549853

The Viscoelastic Behavior of Heat-Set Ovalbumin Gels Explained in Terms of a Transient-Network Model J. Rheol. 29, 685 (1985); 10.1122/1.549806

The World's Most Versatile Platform for Rheological Measurements
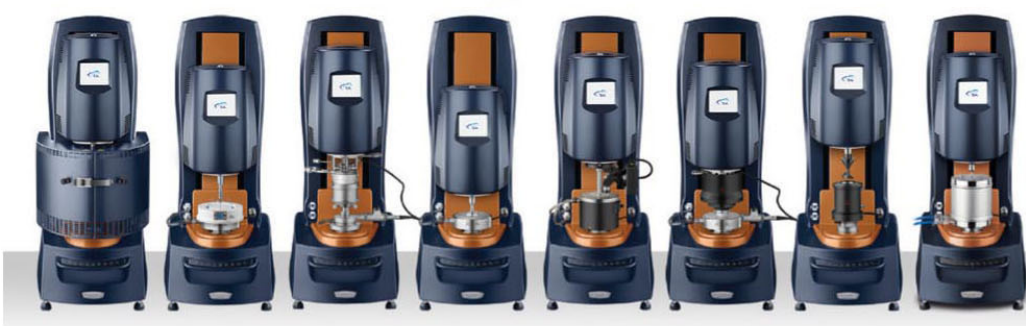

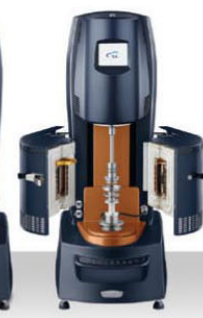

The Discovery

Hybrid Rheometer from

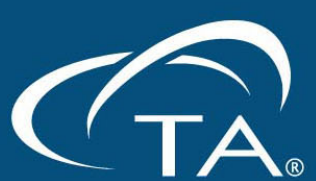

Instruments 


\title{
High bandwidth noninvasive measurements of the linear viscoelasticity of collagen gels
}

\author{
M. Verhulsel \\ Institut Curie, PSL Research University, CNRS, UPMC, UMR 168, F75005 Paris, France \\ M. E. Shivokhin \\ Department of Chemical and Biological Engineering and Center for Molecular Study of Condensed Soft Matter, \\ Illinois Institute of Technology, 3440 S. Dearborn Street, Chicago, Illinois 60616 \\ A. Simon \\ Institut Curie, PSL Research University, CNRS, UMR 144, F75005 Paris, France \\ S. Descroix \\ Institut Curie, PSL Research University, CNRS, UPMC, UMR 168, F75005 Paris, France \\ C. Frétigny and L. Talini ${ }^{\text {a) }}$ \\ ESPCI Paris, PSL Research University, Sciences et Ingénierie de la matière Molle, CNRS UMR7615, 10, \\ Rue Vauquelin, F-75231 Paris Cedex 05, France and Sorbonne-Universités, UPMC Univ. Paris 06, SIMM, 10, \\ Rue Vauquelin, F-75231 Paris Cedex 05, France
}

(Received 10 June 2016; final revision received 16 September 2016; published 24 October 2016)

\begin{abstract}
We report measurements of the linear viscoelastic modulus of collagen gels. Collagen gels are cross-linked networks of bundles formed from the association of single filaments. Their structure is heterogeneous at microscopic length scales, and measurements of their viscoelasticity at a larger scale are required. We use a technique that we recently developed based on the measurement of surface thermal fluctuations and called surface fluctuation specular reflection [Pottier et al., Soft Matter 7, 7843-7850 (2011)] to characterize the linear viscoelastic properties of collagen gels for frequencies ranging from $1 \mathrm{~Hz}$ to $30 \mathrm{kHz}$. These properties are determined at a mesoscopic scale $(\sim 100 \mu \mathrm{m})$ at which the gels are homogeneous. We further study the influence of both collagen origin and concentration in the light of previous findings on these systems. (C) 2016 The Society of Rheology. [http://dx.doi.org/10.1122/1.4965039]
\end{abstract}

\section{INTRODUCTION}

Networks of biopolymers exhibit remarkable mechanical properties, and a large theoretical effort has been devoted to their understanding in the past decades $[1,2]$. Those properties are key parameters of several biological processes, and they make biopolymer networks constitute a new class of materials with seemingly universal features. Unlike flexible polymers and owing to their large persistence length, the dynamics of single chains of biopolymers is governed by bending fluctuations. In addition, the chains can form assemblies, which consist of hundreds of nanometers thick fibrils resulting from the aggregation of several chains, which can themselves bundle into larger structures called fibers. Within the multiscale complex network that is formed, cross links of

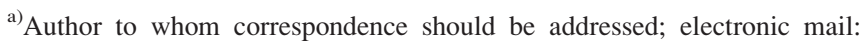
laurence.talini@espci.fr

different natures, permanent or not, originate from the interactions between chains or added linkers. As a result, the networks in particular exhibit strain stiffening which, in vivo, prevents damaging at large strains, and has been recently thoroughly investigated [3,4]. The properties in a linear regime are also of interest since they differ from the ones exhibited by networks of flexible polymers. Different theoretical pictures have been suggested [1,2] some of which account for the nonequilibrium nature of networks of bundles with transient linkers.

Among biopolymer networks, cross-linked gels of F-actin, which is an essential component of cell cytoskeleton, have been widely studied. In contrast, less attention has been paid to the networks formed from collagen. Collagen I-a type of collagen on which we focus herein-is the major component of mammals' extracellular matrix (ECM) [5]. Collagen I molecules spontaneously organize into triple helical structures which further assemble into banded fibrils [6,7]. The organization of banded fibrils networks-i.e., fibrils density, 
alignment, and cross linking-is specific to each tissue in the body [6]. Although collagen I polymerized in vitro does not recapitulate the diversity of living ECM, it successfully replicates the banded fibrils architecture which is the keystone of these matrices [8]. In fact, reproducing in vivo features of matrix ECM is one of the major challenges of tissue engineering as ECM biochemical composition, stiffness as well as its fiber structure, orientation and density can modulate cell adhesion, invasion, migration, proliferation, differentiation, or survival [9]. In this regard, collagen gels are excellent candidates for tissue engineering and it is crucial to better characterize their mechanical properties and, specifically, the link with their microstructure.

However, the determination of the linear viscoelastic properties of collagen gels in a large frequency range is an experimental challenge owing, in particular, to their substantial plasticity resulting from weak cross links [10]. Lately, much effort has been devoted to characterize linear viscoelasticity of collagen gels by non- or only weakly invasive techniques such as optical microscopy $[11,12]$ or microrheology [13-17]. The former techniques are based on the analysis of images of the microscopic structure of collagen networks, and comparison to both rheometer measurements and theoretical predictions for semiflexible polymer networks. Empirical correlations between structure and mechanical properties have been obtained; however, they only provide estimates of the elastic modulus and give no clue on the frequency variations of the complex viscoelastic modulus. Alternatively, several groups have conducted microrheological measurements on collagen gels. Microrheology is based on the motioneither spontaneous or induced —of colloidal probes [18] and provides a quantitative and large bandwidth characterization of linear viscoelasticity with very small strains. However, as other hydrogels [19], collagen gels are networks that are highly heterogeneous at the micrometer scale. It was recently demonstrated that the elastic modulus of collagen gels measured by microrheology could vary by up to one order of magnitude in the same sample according to the location of the micron-sized tracer [16]. One point microrheology is therefore unsuited for those highly heterogeneous gels. In consequence, a technique that is large bandwidth, fully noninvasive, and that probes the material at a length scale larger than its heterogeneities is needed.

In the present work, we characterize the linear viscoelasticity of collagen gels using a noninvasive technique we recently developed, which is based on the measurement of the spontaneous thermal fluctuations of the free surface and called surface fluctuation specular reflection (SFSR) [20]. We analyze experiments conducted at two different length scales, which allows for the determination of the viscoelasticity of materials of unknown surface tension. We first demonstrate the accuracy of the method with two controlled polymeric materials whose moduli are in the same range as collagen gels. We further give evidence for large spatial heterogeneities of collagen gels, confirming previous results [16]. We have performed measurements of the thermal fluctuations of the surface of collagen gels at larger scales at which the gel is homogeneous. We report variations of the viscoelastic modulus of collagen gels over four decades in frequency and discuss them in the light of previous findings. In addition, we emphasize the significant variability of the mechanical properties according to the origin of collagen molecules.

The technique consists of an optical measurement of the spontaneous thermal fluctuations of the free surface, from which bulk viscoelastic properties can be obtained [20]. It is fully noninvasive, and the length scale of the measurement can be tuned from about 1 to $100 \mu \mathrm{m}$. Herein, we show that the fluctuation spectrum of collagen gels is location dependent at smaller scales but not at larger scales, and thus that the linear viscoelastic properties of collagen gels can be determined for frequencies ranging from below $1 \mathrm{~Hz}$ to $30 \mathrm{kHz}$. We have measured the mechanical properties for different concentrations in collagen and analyzed them in the light of the predictions for semiflexible polymers. Section II is devoted to the description of sample preparation, and to the description of the surface fluctuation measurements. The results are presented and discussed in Sec. III.

\section{MATERIALS AND METHODS}

\section{A. Collagen extraction and preparation of solutions}

Collagen samples made from home-extracted collagen and commercial collagen (nonpepsinized rat tail type I collagen, Corning) were used. Unless commercial collagen is specified, we refer to home-extracted collagen in the whole paper. Extraction protocol was adapted from existing protocol [21]. Tendons were pulled out from rat tails and placed in $70 \%$ ethanol to sterilize them. Tendons were separated into thinner strands and transferred to sterile acetic acid $0.2 \%(\mathrm{v} / \mathrm{v})$ at $4{ }^{\circ} \mathrm{C}$ for 5 days. The collagen extract was first centrifuged at $8800 \mathrm{~g}$ at $4{ }^{\circ} \mathrm{C}$ for $30 \mathrm{~min}$ to discard the nondissolved bundles. The supernatant was again centrifuged for $90 \mathrm{~min}$ at $4{ }^{\circ} \mathrm{C}$ at $8800 \mathrm{~g}$. The top clear collagen solution was lyophilized, and dried collagen was stored at $-20^{\circ} \mathrm{C}$. To prepare collagen stock solutions, dried collagen was weighted and resuspended in $0.2 \%(\mathrm{v} / \mathrm{v})$ acetic acid at a concentration of $20 \mathrm{mg} \mathrm{ml}^{-1}$. To ensure its homogeneity, a collagen stock solution was manually vigorously shaken for $30 \mathrm{~s}$ per day during 5 days. After 5 days, the whole collagen was completely dissolved, and the obtained solution was translucent attesting of its homogeneity. Stock solution was then kept at $4{ }^{\circ} \mathrm{C}$. To observe the fibrillar structure of collagen gels with confocal microscopy, we labeled collagen in a solution with Tetramethylrhodamine (TAMRA, Invitrogen), as detailed elsewhere [22,23]. Briefly, collagen I from stock solution was dialyzed overnight against labeling buffer $\left(0.25 \mathrm{M} \mathrm{NaHCO}_{3}, 0.4 \mathrm{NaCl}, p \mathrm{H} 9.5\right)$ at $4{ }^{\circ} \mathrm{C}$. Tetramethyl-rhodamine (TAMRA, Invitrogen) was dissolved in dimethylsulfoxyde (DMSO) according to supplier's instructions. TAMRA in DMSO was then diluted to $10 \%(\mathrm{v} / \mathrm{v})$ in labeling buffer and mixed with dialyzed collagen (1:1 in ratio). TAMRA-collagen solution was incubated overnight under constant agitation. TAMRA-collagen solution was then dialyzed overnight against labeling buffer to remove free dye. A final overnight dialysis in acetic acid $0.2 \%(\mathrm{v} / \mathrm{v})$ of labeled collagen solution was performed. 
Investigated final collagen concentrations were 2, 4, 6, and $10 \mathrm{mg} \mathrm{ml}^{-1}$. Soft collagen gels $\left(2-4 \mathrm{mg} \mathrm{ml}^{-1}\right)$, easily remodeled by cells, are preferentially used to study cell invasion and migration while highly concentrated collagen gels appear as a great model to question phenotypic changes in the context of fibrosis [24]. The volume of collagen needed was placed in an eppendorf tube kept on ice using a pipette dedicated to viscous liquids (Transferpettor, $500 \mu \mathrm{l}$, BrandTech). The neutralizing collagen solution which allows collagen polymerization was prepared in another tube also kept on ice. Neutralizing reagents consist of 10X phosphate buffered saline (PBS) (Gibco), cell culture medium (DMEM glutamax, Gibco), and $1 \mathrm{~N} \mathrm{NaOH}$. The relative amount of each constituent was calculated as follows:

$$
V_{P B S}=0.1 V_{\text {final }} \text {. }
$$

$V_{\mathrm{NaOH}}=0.022 V_{\text {Collagen }}$ for commercial collagen (nonpepsinized, Corning) and $0.04 V_{\text {Collagen }}$ for home-made collagen and TAMRA labeled collagen

$$
V_{D M E M}=V_{\text {Final }}-V_{P B S}-V_{\text {Collagen }}-V_{\mathrm{NaOH}} .
$$

The neutralizing solution was mixed on ice and pipetted on the top of collagen before injection and polymerization in the polydimethylsiloxane (PDMS) holder. Special care was taken to avoid bubbles in the gel. The $p \mathrm{H}$ of the solution was controlled to be at 7.4 which is consistent with cell culture. Fluorescent collagen gels were obtained by mixing $10 \%$ w/ w TAMRA labeled collagen to nonlabeled collagen. Images of fluorescent gels were collected after collagen polymerization using an inverted laser scanning confocal microscope (LSM710NLO Zeiss, x40/1,3 OIL DICII PL APO (UV) VISIR (4020762-9800)). Pictures were taken $60 \mu \mathrm{m}$ above coverslips.

\section{B. Fabrication of flat collagen gels}

PDMS (Sylgard 184, Dow Corning) was mixed with $10 \% \mathrm{w} / \mathrm{w}$ curing agent and cured at $60^{\circ} \mathrm{C}$ for $4 \mathrm{~h}$ to obtain a flat layer. PDMS washers of $1 \mathrm{~cm}$ inner diameter and $2 \mathrm{~cm}$ outer diameter and $0.5 \mathrm{~cm}$ height were punched out of this PDMS. Two holes (one injection and one outlet, $3 \mathrm{~mm}$ diameter) were punched in the walls of PDMS washers. Washers were then placed between two glass slides to form an enclosed chamber. The liquid collagen solution was injected in the enclosed chamber using a $2 \mathrm{ml}$ syringe with a $1 \mathrm{ml}$ cut cone placed at its tip. Once the chamber was filled, collagen solution was left to polymerize at room temperature for 30 min. Collagen polymerization in an enclosed chamber ensured the flatness of the surface of the gel and avoided any dehydration of the gel while polymerizing.

\section{Other cross-linked polymeric materials}

In order to validate the linear viscoelastic measurements conducted with the SFSR technique, two cross-linked polymeric materials were used: a permanently cross-linked PDMS (Sylgard 186, Dow Corning) and gels formed from solutions of associating polymers (terpyridine-functionalized methacryl-succinimidyl
(MASI) modified supramolecular poly(N-isopropylacrylamide (pNIPAAm) in Dimethylformamide (DMF)) [25,26]. The polymer concentration was $20 \mathrm{wt} \%$ and addition of $\mathrm{Mn}\left(\mathrm{NO}_{3}\right)_{2}$ ions at a stoichiometric ratio of 2.0 allowed for formation of transient cross links between chains. Both SFSR and rheometry experiments were conducted on those materials.

\section{Rheometric experiments}

Oscillatory measurements were conducted with both pNIPAAM gel and cross-linked PDMS. For the pNIPAAM gel, a TA Instruments advanced rheometric expansion system (ARES) equipped with a cone-plate fixture of $25 \mathrm{~mm}$ was used. The sample was equilibrated at $25^{\circ} \mathrm{C}$ for approximately $30 \mathrm{~min}$ prior to the frequency sweep measurement. Measurements with PDMS were conducted on an Anton Paar MCR 501 rheometer in a simple shear strain, with plate geometry. The temperature was varied from -30 to $+30^{\circ} \mathrm{C}$ every $3{ }^{\circ} \mathrm{C}$. A frequency sweep between 0.01 and $50 \mathrm{~Hz}$ was performed at each temperature, and the elastic and loss moduli were further deduced in the range of $0.1-10^{4} \mathrm{~Hz}$ using time-temperature superposition.

\section{E. Surface fluctuation measurements}

The collagen samples were unmolded and placed into a cell filled with PBS such that only the upper surface of the collagen gel was not covered with PBS. After an equilibration time of about one hour, surface fluctuation measurements were performed. No evolution of the sample was monitored during the experiments, and a test measurement carried out $24 \mathrm{~h}$ after placing the sample in the cell showed no significant modification of the sample at all investigated scales, ensuring dehydration is negligible during an experiment. In addition, the result evidences that the gel does not age over the timescale of an experiment, unlike other biopolymer networks [27].

No specific care was taken with the polymeric materials used for validation of the new development of SFSR with two beam sizes (detailed in Sec. III): a parallelepipedic piece of centimetric dimensions was cut in the cured PDMS sample, and $1.5 \mathrm{ml}$ of the pNIPAAm solution was placed in a cell of diameter $2.5 \mathrm{~cm}$.

As schematized in Fig. 1, SFSR uses the reflection of a low power $(1 \mathrm{~mW})$ laser at the free surface of the sample. Spontaneous thermal fluctuations of the surface result in fluctuations of the position of the reflected laser beam. The latter fluctuations are measured with a two quadrant photodiode on which the reflected beam is centered. The light intensity difference between the two quadrants results in a current difference which is converted into voltage, amplified, antialiasing filtered and further digitized. The laser is focused at the surface with a converging lens or a microscope objective according to the desired beam radius $R$ at the surface. Three different optics yielding three different beam sizes were used: a microscope objective $(\times 20)$ yielding $2 R=7 \mu \mathrm{m}$, a $6 \mathrm{~cm}$ focal length lens yielding $2 R=55 \mu \mathrm{m}$, and a $9 \mathrm{~cm}$ focal length lens yielding $2 R=83 \mu \mathrm{m}$. The radii were determined from preliminary measurements performed with liquids of known viscosities and surface tensions (silicon oils). 


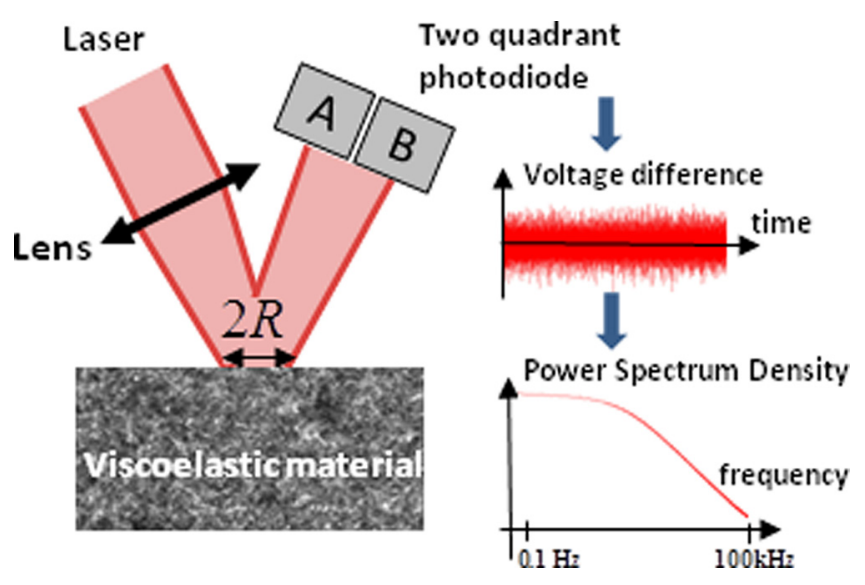

FIG. 1. Principle of the SFSR measurement of linear viscoelastic properties. A laser beam is focused at the free surface of a viscoelastic material, and the reflected beam is centered on the two quadrants of a photodiode. Thermal fluctuations of the free surface result in light intensity fluctuations on the two quadrants, which are converted into a voltage difference recorded as a function of time. The power spectrum density of that noise is computed for further analysis in order to obtain the frequency dependent linear viscoelastic modulus of the material. The beam size at the surface, $2 R$, sets the characteristic length scale of the measurement.

The power spectrum density $S(\omega)$ of the time signal is computed in order to analyze the frequency content of the measured noise. More experimental details can be found elsewhere [28]. $S(\omega)$ depends on both the probed material properties and laser beam characteristics. More precisely, we have shown that for a viscoelastic material of complex viscoelastic modulus $G^{*}(\omega), S(\omega)$ could be expressed as a function of the material properties, namely, the modulus $G^{*}(\omega)$, the density $\rho$, and the surface tension with air $\sigma$. In addition, $S(\omega)$ depends on the laser beam properties, namely, its divergence and its radius at the surface, the latter setting the length scale of the measurement, i.e., the characteristic wavelength of the spatial modes that contribute most to the signal. The beam wavelength $\lambda$ and radius $R$ are therefore additional parameters in the expression for $S(\omega)$ [20]

$$
S(\omega)=B \frac{k_{B} T}{\lambda^{2} \pi^{3} \omega} \int_{0}^{\infty} \operatorname{Im}(\chi(\omega, k)) \exp \left(-\frac{k^{2} R^{2}}{c}\right) k^{3} R^{2} d k,
$$

where Im denotes the imaginary part, $k_{B} T$ is the thermal energy, and $B$ and $c$ are numerical constants that result from a numerical approximation [28]. The mechanical susceptibility $\chi(\omega, k)$ is a function of the material properties such that $[28]$

$$
\begin{aligned}
\frac{1}{\chi(\omega, k)}= & \sigma k^{2}+G^{* 2}(\omega) \frac{k^{3}}{\omega^{2} \rho} \\
& \times\left[4 \sqrt{1-\frac{\omega^{2} \rho}{k^{2} G^{*}(\omega)}}-\left(2-\frac{\omega^{2} \rho}{k^{2} G^{*}(\omega)}\right)^{2}\right] .
\end{aligned}
$$

Provided all other parameters are known, the frequency dependent elastic and loss moduli of the material can be inferred from the measured $S(\omega)$. In practice, a fit to the experimental values of $S(\omega)$ is performed in order to determine both the real and imaginary parts $G^{\prime}(\omega)$ and $G^{\prime \prime}(\omega)$. A Kramer-Kronig relation is implicitly used by writing the complex viscoelastic modulus in the frame of a KramersKronig compliant model, for instance, a generalized Maxwell model which is written as

$$
G^{*}(\omega)=\sum_{k=1}^{N} \frac{H_{k} i \omega \tau_{k}}{1+i \omega \tau_{k}},
$$

where $i^{2}=-1$, and $\tau_{k}$ and $H_{k}$, respectively, denote the relaxation time and amplitude of the kth Maxwell mode.

We have found that a viscoelastic modulus can generally be described using three relaxation times per time decade [20]. Therefore, in the fitting procedure of the fluctuation spectra, the relaxation time basis is set and the fitting parameters are the amplitudes $H_{k}$ of the Maxwell modes. Once the amplitudes $H_{k}$ are determined, the real and imaginary part of the right term of Eq. (3) are computed in order to obtain, respectively, the frequency dependent elastic modulus $G^{\prime}(\omega)$ and loss modulus $G^{\prime \prime}(\omega)$. We emphasize that the obtained moduli do not depend on the model chosen to describe their frequency variations in the fitting process: any rheological model satisfying the Kramers-Kronig relations would yield the same moduli. The fitting process is an alternative to the direct use of the Kramers-Kronig relations, which requires computations of Hilbert transforms that are numerically delicate. We have shown in the past works [20,29] that SFSR provides a useful tool to characterize the linear viscoelasticity of soft materials provided their surface tension is known. In the following, we show that, by performing measurements with two laser beam sizes, it is possible to determine the linear viscoelasticity of soft materials without conducting independent measurements of their surface tension.

\section{RESULTS AND DISCUSSION}

\section{A. Spatial heterogeneities}

Measurements of surface fluctuations at different length scales were performed on collagen gels. As mentioned earlier, the laser beam size determines the characteristic wavelength of the spatial modes that mostly contribute to the signal, and thus gives the length scale of the measurement. At smallest beam size $(2 R=7 \mu \mathrm{m})$, we have found that the fluctuation spectra of a collagen gel of concentration $4 \mathrm{mg} \mathrm{ml}^{-1}$ depend on the location of the beam at its free surface [Fig. 2(a)]. That dependence reveals spatial heterogeneities at that scale, resulting in heterogeneous properties. Although they appear rather small in the logarithmic scale of Fig. 2(a), the differences between the spectra are significant and correspond to the differences in elastic or loss modulus larger than $30 \%$, as shown in Fig. 2(c).

In contrast, spectra measured at the surface of the same collagen sample with a larger beam size $(2 R=55 \mu \mathrm{m})$ coincide whatever the beam location at the surface: in Fig. 2(b), the spectra obtained at three different locations superimpose. At that scale, the gel is homogeneous and its linear viscoelastic properties can be derived from the fluctuation spectra. We emphasize that the measured mechanical properties correspond to bulk properties of the material. They are actually 

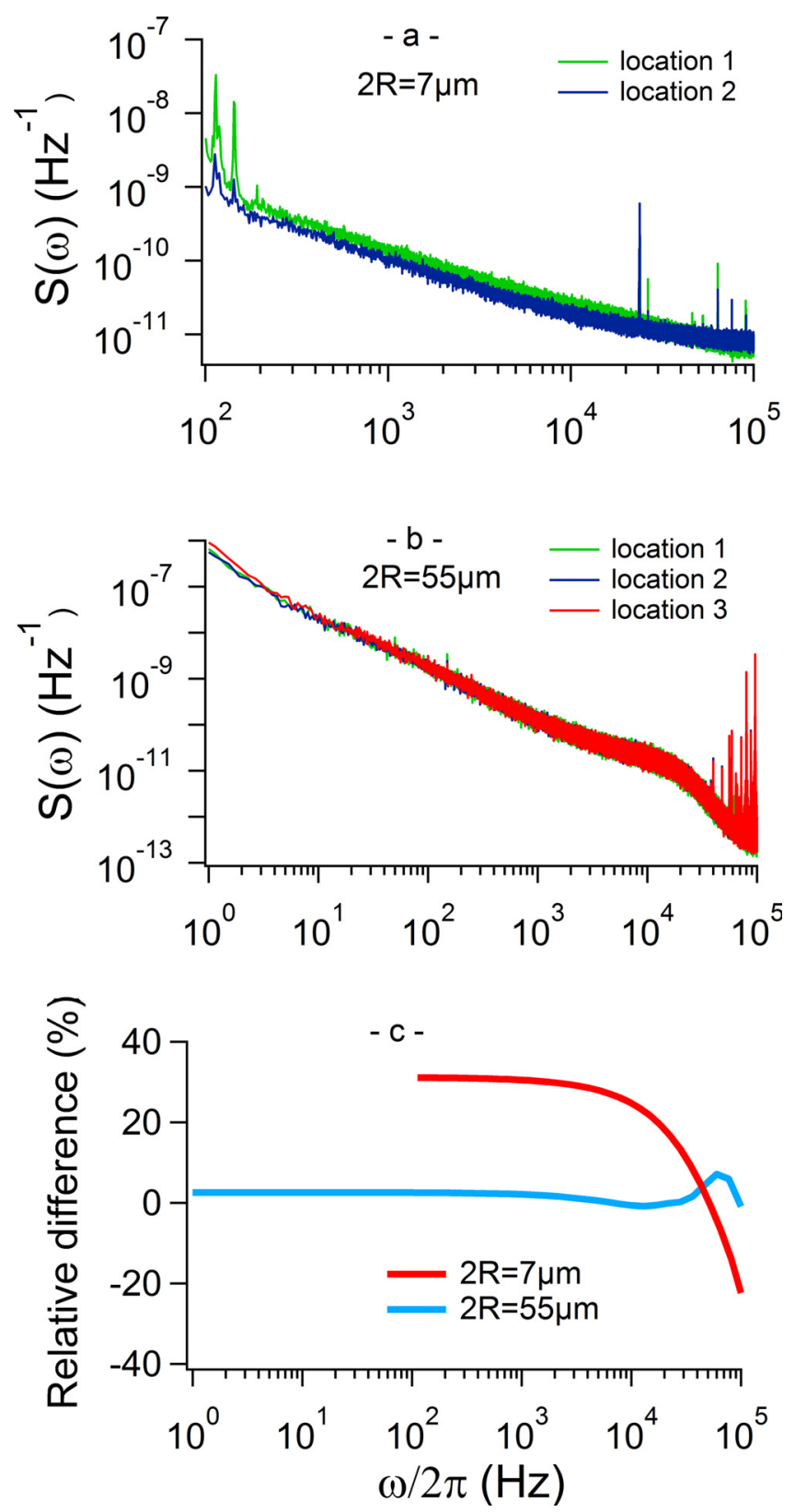

FIG. 2. Fluctuation spectra measured at different locations of the same collagen gel (concentration $4 \mathrm{mg} \mathrm{ml}^{-1}$ ) with beam size $2 R=7 \mu \mathrm{m}$ (a) and $2 R=55 \mu \mathrm{m}$ (b). In (a), the differences in the spectra result from spatial heterogeneities of the gel at the probed scale, whereas the location independent spectra in (b) reveal homogeneous properties at the larger scale. In (c), the relative differences between spectra at two different locations are shown for the same probed scales as in, respectively, (a) and (b). They correspond to the ratio of the difference of spectra at two locations and the average spectrum, which have been further interpolated for the sake of clarity.

the average properties of a volume scaling as $R^{3}$ with vertical extent scaling as $R$, since the radius beam determines the length scale of the measurement.

Confocal microscopy images (Fig. 3) confirm the highly heterogeneous structure of the gels at the micron scale. Collagen is known to form branched network structures with pore size scaling as $c^{-0.5}$ [12], and the less concentrated samples are therefore expected to be more heterogeneous, consistently with our observations.
We have found that gels made from commercial collagen exhibit heterogeneous structures at a scale larger than gels prepared from home-made collagen at the same concentration. For the less concentrated commercial collagen samples ( 2 and $4 \mathrm{mg} \mathrm{ml}^{-1}$ ), spatial heterogeneities were measured with a beam size $2 R=55 \mu \mathrm{m}$, whereas the home-made collagen gels are homogeneous at that scale. Consistently, qualitative differences in the structure of those gels can be observed in confocal microscopy images (Fig. 3). Observations of the spatial heterogeneities of collagen gels have been reported in the literature [16].

In particular, microrheological measurements performed with micron-sized tracers have revealed highly heterogeneous gels [16]. More generally, hydrogels are heterogeneous materials.

For instance, micron-sized heterogeneities were measured in polyethylene glycol hydrogels from indentation experiments with colloidal probes [19]. As a result, a careful examination of sample heterogeneities is needed before any measurement of the linear viscoelastic properties of collagen gels. Our data furthermore confirm that the use of colloidal probes (in either microrheology or indentation) is not suited for characterizing the mechanical properties of collagen gels, whereas the more mesoscopic and tunable length scale of SFSR makes it well adapted for those media. Among other available techniques, two point-microrheology [30] could also be used to characterize the linear viscoelasticity of collagen gels, although no reports of such measurements are found in the literature. The technique uses the cross correlation of the Brownian motions of two colloidal tracers and thus probes the properties at length scales given by the distance between tracers and not by their size. However, it requires the addition of tracers and, therefore, it is not fully noninvasive as the SFSR technique.

\section{B. Measurement of linear viscoelastic properties}

We show in what follows that the linear viscoelastic modulus of collagen gels can be obtained from homogeneous SFSR measurements, over a large frequency range. For soft viscoelastic materials, the dynamics of surface fluctuations, in particular, depends on both surface tension and elasticity, which are the restoring effects at stake when the free surface is deformed. For a spatial mode of wavelength $R$, the critical value of the viscoelastic modulus is given by $\sigma / R$ that sets the low-frequency limit between regimes dominated by surface tension effects (below that value) and elasticity (above that value) [20]. For collagen gels and a beam size $2 R=55 \mu \mathrm{m}$, $\sigma / R$ is of a few $\mathrm{kPa}$, which is precisely the expected order of magnitude of the elastic modulus. An accurate determination of the viscoelastic modulus from fluctuation spectra may therefore require the value of surface tension with air of the gel. More precisely, the elastic modulus determined from the fluctuation spectra may strongly depend on the chosen values of surface tension. In contrast, the loss modulus is expected to only weakly depend on that value [29]. The surface tensions of collagen gels are not known a priori. Recently, evidence has been given that the surface tension of gels of hydrosoluble polymers may be significantly smaller than the surface tension 

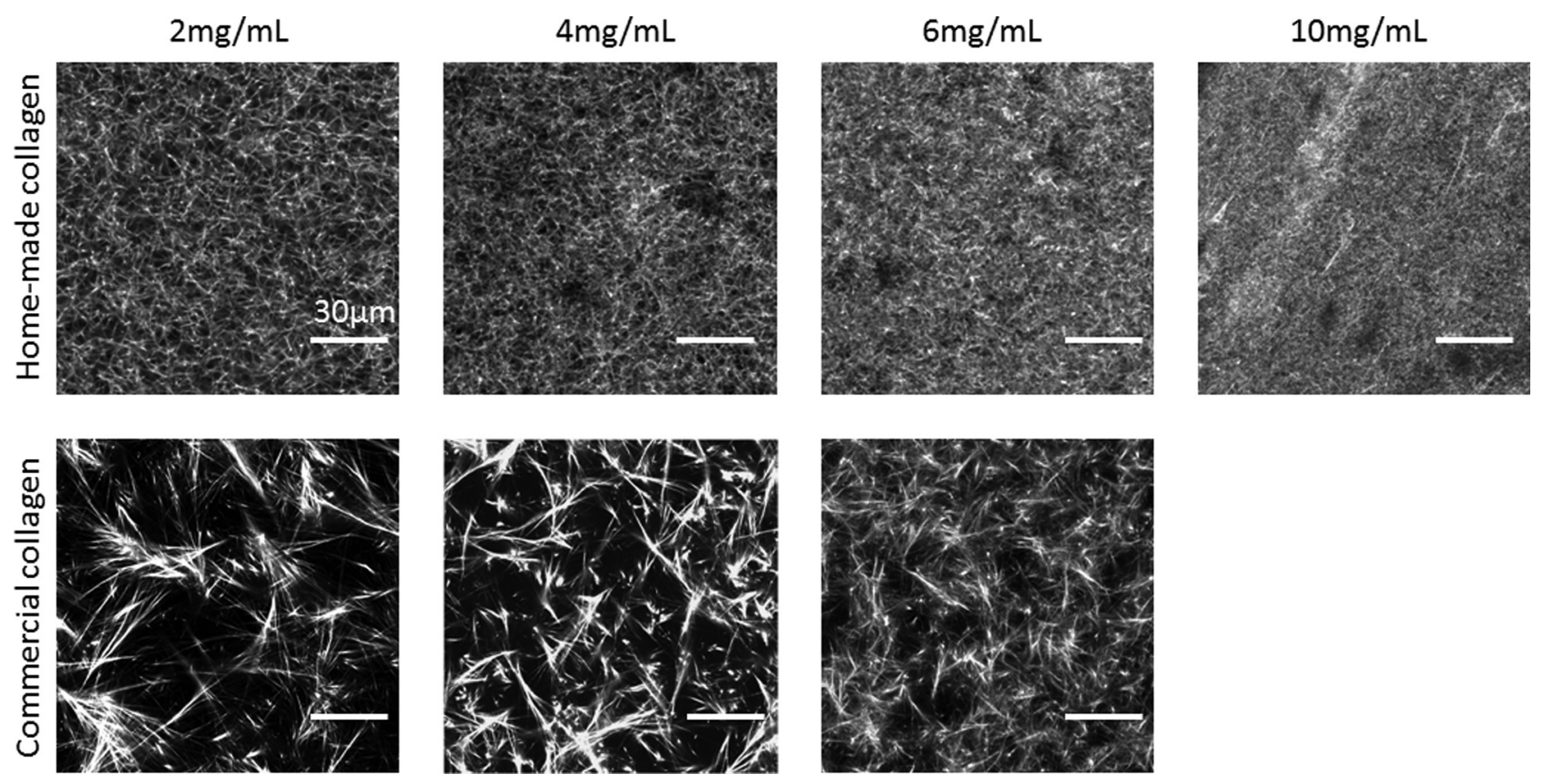

FIG. 3. Fluorescent images of commercial and home-made collagen gels at different concentrations. Same scale in all images.

of water with air, with values down to about $50 \mathrm{mN} \mathrm{m}^{-1}$ [31]. That effect is likely to result from adsorption of polymers at the air/gel interface, combined to air exposure of the more hydrophobic parts of the polymers, as observed, for instance, in wetting experiments of polymeric substrates [32]. Consequently, surface tension is expected to depend on polymer concentration. The determination of surface tension of gels with air is not straightforward, and there are only a few existing techniques [31].

Instead of conducting independent measurements of surface tension, we have determined both the viscoelastic modulus and the surface tension of each sample from surface fluctuation spectra. We have thus performed successive experiments with two different laser beam radii at the surface of the same sample. As mentioned earlier, the beam radius determines the wavelengths of the spatial modes that are mainly probed. As a result, the spectra obtained with different radii on the same sample have different amplitudes and shapes and contain the necessary information to fully determine both surface tension and viscoelastic modulus.

In order to validate that new development of SFSR technique, we have first performed experiments with the polymeric systems described in the experimental section, namely, a permanently cross-linked PDMS and a modified associative pNIPAAM in solution with transient cross links. Those systems were chosen because their elastic moduli are, respectively, of the order of 10 and $1 \mathrm{kPa}$, which corresponds to the modulus range of collagen gels at the concentrations we probe. Figure 4 displays the spectra, respectively, obtained with beam size $2 R=55 \mu \mathrm{m}$ and $2 R=83 \mu \mathrm{m}$ with the pNIPAAM solution. Surface tension $\sigma$ and the complex viscoelastic modulus were concomitantly determined by performing a single fit to both experimental spectra. As detailed above, the viscoelastic modulus is written in the frame of a generalized Maxwell model following Eq. (3). The fitting parameters are the amplitudes $H_{k}$ of the Maxwell modes together with surface tension, on which values the spectra depend following Eqs. (1) and (2). All other parameters appearing in Eqs. (1) and (2) are known and kept constant during the fitting process.

The value found for surface tension of the pNIPAAM solution is the one of its solvent $\left(37 \mathrm{Nm}^{-1}\right.$ for DMF), which is expected if the polymers do not absorb at the interface with air. The viscoelastic moduli of the solution determined by the same fitting procedure as surface tension are shown in Fig. 4(b); they are in good agreement with the rheometric data within the common frequency range of rheometry and SFSR. A similar agreement was found with cross-linked PDMS for which time-temperature superposition allows for the measurements of rheometric data in a wider frequency range.

We have found that an accurate value for surface tension is needed to correctly determine the viscoelastic properties of the pNIPAAM solution, as expected when the magnitudes of capillary and elastic effects are of the same order. In contrast, elastic effects dominate at the surface of the more rigid cross-linked PDMS, and it is rather the value of the zero frequency elastic modulus that is needed to unambiguously determine the viscoelastic properties for that material, in agreement with previous findings [20]. That value is provided by the fitting process to the data obtained with two beam sizes. We therefore claim that the new development of SFSR we present allows for the full determination of the viscoelastic properties of soft materials from about $1 \mathrm{~Hz}$ to $10 \mathrm{kHz}$, without prior determination of the surface tension nor the zero frequency modulus.

SFSR measurements were conducted with the collagen samples. SFSR measurements were made at scales at which the gels are homogeneous: beam sizes $2 R=55 \mu \mathrm{m}$ and $2 R$ $=83 \mu \mathrm{m}$ were used. Homogeneity was first checked on each sample by comparing the fluctuation spectra obtained at different locations. Spectra at a given location were further obtained for the two beam sizes. Two-spectra fitting processes were successfully performed on the experimental data. The 

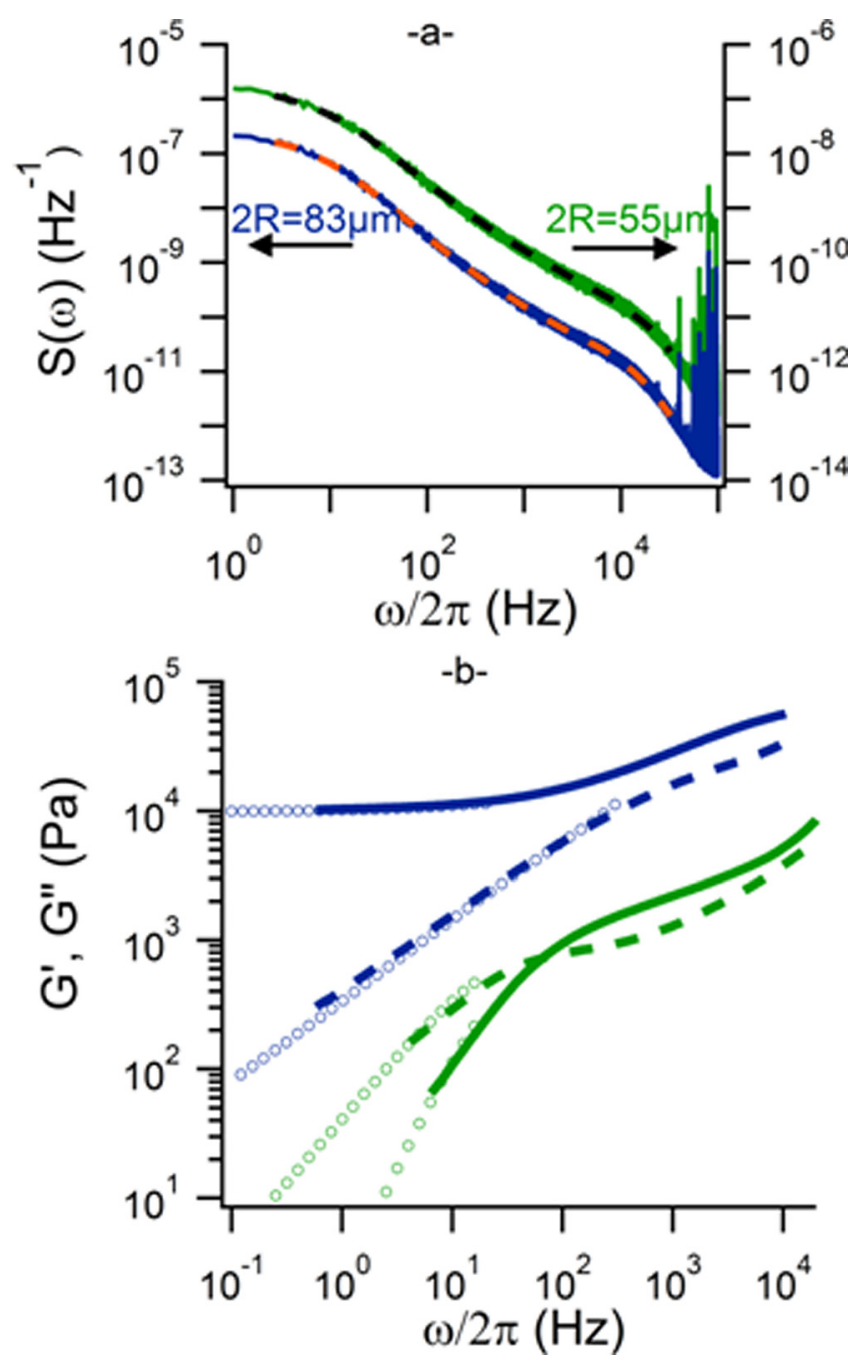

FIG. 4. (a) Fluctuation spectra of the same modified pNIPAAm solution, with two different laser beam radii: $2 R=83 \mu \mathrm{m}$ (left axis) and $2 R=55 \mu \mathrm{m}$ (right axis). The beam size sets the wavelength of the spatial modes that mostly contribute to the signal, leading to different spectra for the same sample. The dotted lines are the best fitting curves obtained from the simultaneous two-spectra fitting procedure described in the text. The large peaks at high frequency result from parasitic noise of the experimental setup. (b) Frequency-dependent elastic (full lines) and loss moduli (dotted lines) obtained from the SFSR measurements with the pNIPAAm solution (green/ lighter lines) and the cross-linked PDMS (blue/darker lines). Rheometry measurements were also conducted for each sample (circles) at room temperature for the pNIPAAm solution, and at temperatures ranging from $-30^{\circ} \mathrm{C}$ to $30^{\circ} \mathrm{C}$ for the PDMS. For the latter, time-temperature superposition was used to cover a wider frequency range for the master curve at the reference temperature $\left(20^{\circ} \mathrm{C}\right)$.

found values for surface tension are, respectively, $60,52,48$, and $44 \mathrm{mN} \mathrm{m}^{-1}$ for concentrations of 2, 4, 6, and $10 \mathrm{mg} / \mathrm{ml}$. In agreement with results from the literature obtained on other hydrogels [26], they are significantly smaller than the surface tension of water with air and decrease with increasing collagen concentration.

\section{Frequency dependent viscoelastic modulus of collagen gels}

The fluctuation spectra measured with two beam radii and the elastic and loss moduli inferred from their fitting are shown respectively in Figs. 5 and 6 for a home-made collagen sample

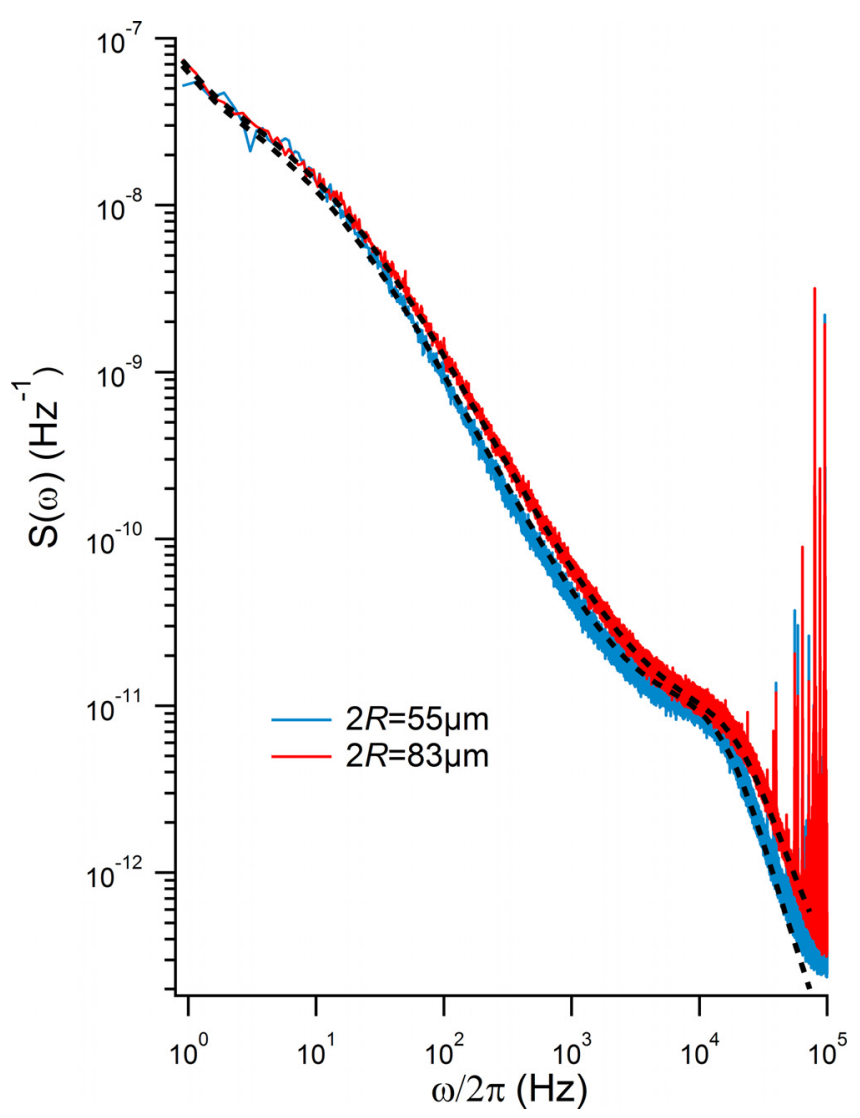

FIG. 5. Fluctuation spectra of the same sample (collagen concentration $6 \mathrm{mg} \cdot \mathrm{mL}^{-1}$ ), with two different laser beam radii. The beam size sets the length scale of the measurement (i.e., the wavelength of the spatial modes that mainly contribute to the signal), and as a result, the spectra differ although they correspond to the same sample, which is homogeneous at the probed scale. The dotted black lines are the best fitting curves obtained from the simultaneous fitting procedure described in the text. The large peaks at high frequency result from parasitic noise of the experimental setup.

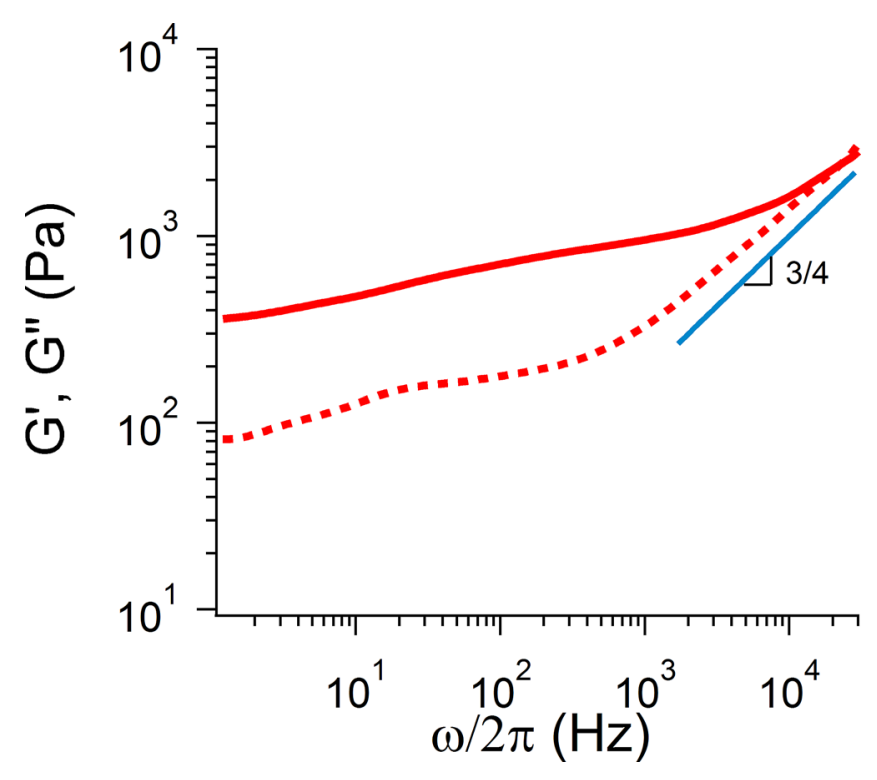

FIG. 6. Elastic (full line) and loss (dotted line) moduli of a home-made collagen gel of concentration $4 \mathrm{mg} \mathrm{ml}^{-1}$. The data were obtained from two beam fitting of fluctuation spectra. The corresponding value of surface tension is $52 \mathrm{mN} \mathrm{m}^{-1}$. The solid blue line represents a power law with an exponent $\frac{3}{4}$. 
of concentration $c=4 \mathrm{mg} \mathrm{ml}^{-1}$. Over more than four decades in frequency are covered, from about $1 \mathrm{~Hz}$ to $30 \mathrm{kHz}$. At lower frequencies, the moduli increase weakly with increasing frequency. At larger frequencies, the slope becomes steeper for both moduli. Similar variations have been reported in other biopolymer networks. In particular, measurements made on the actin networks of the cytoskeleton exhibit striking similarities [33].

The high frequency moduli of single semiflexible filaments have been shown to increase with frequency to the power $\frac{3}{4}$ [34,35]. In the networks we consider, one expects to recover the single filament dynamics at high frequency, which was previously reported in different biopolymer networks or solutions $[33,36]$. At smaller frequencies, the elastic modulus is predicted to plateau for permanently cross-linked networks of single filaments. In contrast, a slow increase of the elastic modulus with increasing frequency is observed in bundle networks; the increase is generally described by a power law with an exponent close to 0.1 [33]. Consistently, we report a weak increase of the modulus of collagen gels at small frequencies, followed by a stronger increase at large frequencies.

At small frequencies, a power law with an exponent close to 0.2 successfully describes the variations of the elastic modulus. In networks of bundles such as collagen gels, the high concentration and transitory nature of cross links modify the rheological properties and, in particular, the elastic plateau. Evidence has been given that those networks are in a nonequilibrium state, meaning that cross links slow down the relaxations of the filaments such that the equilibrium state is never reached [37].

A qualitative agreement with the latter behavior was recently reported from finite element simulations of transient bundle networks [27]. Semiflexible filaments could link via chemical reactions with fixed rate constants that were chosen close to the ones of F-actin protein linkers. The same slow increase with frequency of the elastic modulus was observed in the intermediate frequency range. It was attributed to the destructive effect of strain on bonds between filaments, even though the probed regime was linear, because the overall network remained intact. In contrast, the expected $\omega^{3 / 4}$ power law at high frequency was not recovered in the simulations. Instead, different exponents were found at those frequencies for the elastic and loss modulus (of values close to, respectively, 0.5 and 1). The high frequency behavior was attributed to a length scale-dependent bending modulus for bundle, in contrast to the constant bending modulus of single filaments.

Statements on the frequency behavior are however difficult to make from our experimental data. Although they span more than four decades in frequency, both very low and high frequency data would be needed to ascertain the existence of a true elastic plateau and the exponent characterizing the increase at high frequency. At low frequency, parasitic noise mainly resulting from vibrations hinders surface thermal fluctuations and renders measurements difficult. Measurements at frequencies larger than $30 \mathrm{kHz}$ are also challenging. In that range, SFSR is limited by the noise of the experimental setup mainly originating from the current to voltage converter of the photodiode. Experiments conducted with smaller beam size would increase the signal to noise ratio at large frequencies but are not suited for the heterogeneous collagen gels. We can nevertheless infer from the obtained variations that cross links modify the dynamics down to very small scales, since their effect is observed at frequencies as large as a few $\mathrm{kHz}$.

In summary, we report, for the first time to our knowledge, a determination of the frequency variations of collagen gels over a very large frequency range. The viscoelastic modulus was found to behave similarly as in other networks formed by bundles of biopolymers. In particular, we have observed a slow increase at small frequencies of the moduli of collagen gels. In Sec. III D, we study how the viscoelastic modulus varies with collagen origin and concentration.

\section{Dependence of the viscoelastic properties on the origin of collagen and concentration}

In Fig. 7, we report the variation with concentration of the elastic modulus of home-extracted collagen gels for frequencies of 10 and $100 \mathrm{~Hz}$. The values show a quantitative agreement with recent values obtained by rheometry for smaller concentrations [4]. A power law increase with frequency of the elastic modulus was found for concentrations ranging from 0.5 to $3.5 \mathrm{mg} \mathrm{ml}^{-1}$, with an exponent close to 2 . Consistently, the SFSR data is well described by a power law with an exponent of very similar value. Our data confirm the previous results and furthermore provide new information on highly concentrated collagen gels, which have been poorly studied up to date.

A power law with an exponent of $11 / 5$ is predicted for the concentration dependence of the plateau modulus of permanently cross-linked networks of semiflexible filaments; the same power law was found in numerical simulations of bundle networks with transient cross linking from [27]. In

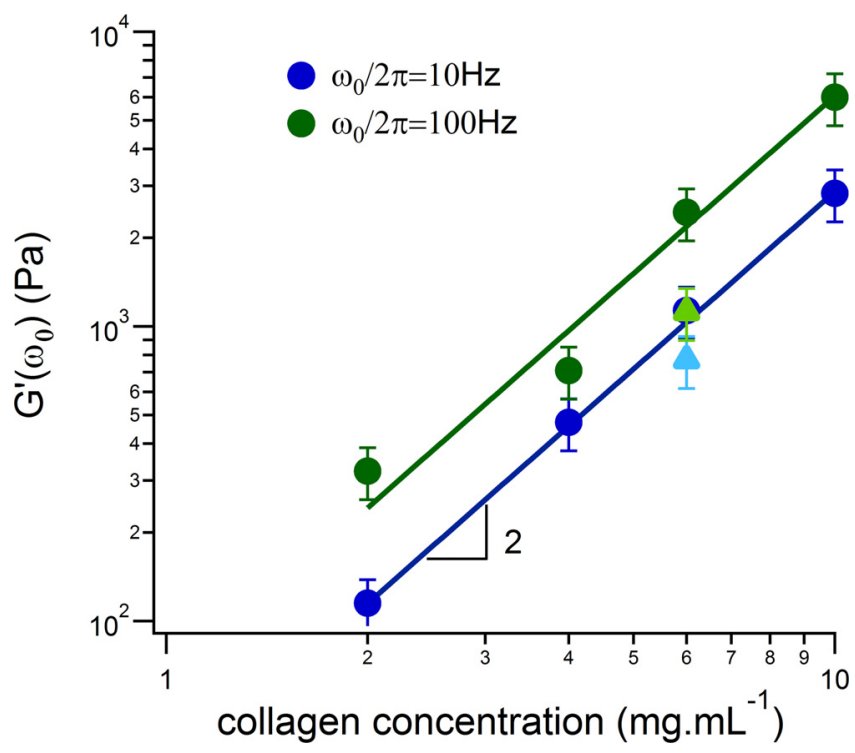

FIG. 7. Elastic modulus of collagen gels as a function of collagen concentration measured at two frequencies. All data correspond to home-made collagen except the triangles that correspond to commercial collagen. All samples were found to be homogeneous with the two beam sizes used $(2 R=55 \mu \mathrm{m}$ and $2 R=83 \mu \mathrm{m})$. The full lines represent power laws with exponent 2 . The error bars indicate the variability on the modulus for different collagen samples prepared at the same concentration. 
contrast, for athermal networks such as the ones formed by collagen fibers, an exponent of 2 has been predicted [2]. Our data are consistent with the last results although experimental data do not allow discrimination between models.

Finally, we have found the mechanical properties of gels formed from either home-extracted or commercial collagen molecules significantly differ. As mentioned earlier, the gels prepared with commercial collagen form structures that are heterogeneous at a larger scale than the gels prepared from homemade collagen. However, at a concentration of $6 \mathrm{mg} \mathrm{ml}^{-1}$, the samples were found to be homogeneous and the viscoelastic properties of commercial collagen gels could be measured. The moduli of those samples are smaller by a factor ranging from 1.5 to 2 than the ones of home-extracted samples, as shown in Fig. 7. That result evidences the difficulty to quantitatively compare collagen gels of different origins. In addition, it could provide an explanation for the very different values found in the literature for the elastic modulus of collagen gels. In the case of the two collagens we consider herein, the differences could result from small differences in their chemical nature [38].

\section{CONCLUSION}

In conclusion, we have successfully used the thermal fluctuations of the free surface of collagen gels to characterize their linear viscoelasticity. The versatile length scale of the measurement allows for characterization at a scale at which the gels are homogeneous, which we have shown to be mesoscopic. The fluctuation spectra depend on both the gel surface tension with air and viscoelastic modulus; using well controlled crosslinked materials, we have shown that these parameters can be unambiguously determined by analyzing measurements conducted at two different length scales. In particular, the obtained viscoelastic modulus is in excellent agreement with rheometric data. We have thus measured the frequency dependent elastic and loss moduli of gels whose collagen concentration ranges from 2 to $10 \mathrm{mg} \mathrm{ml}^{-1}$, and spanning over four decades in frequency. The found variations are qualitatively similar to the ones for other biopolymer networks with transient cross linking. A slow increase of the moduli is observed, with a slope increase at larger frequencies. The scaling with concentration of the elastic modulus at smaller frequencies is in good agreement with the predictions for cross-linked networks of bundles, according to the previous reports in the literature. We also demonstrate that samples made from commercial and homeextracted collagen can present significant differences in sample homogeneity and viscoelastic properties. We emphasize that the SFSR technique provides a new fully noninvasive method to characterize the linear viscoelasticity of highly heterogeneous soft materials.

\section{ACKNOWLEDGMENTS}

The authors gratefully acknowledge the Fondation Langlois for partial funding, and PICT-IBiSA Imaging Facility, Institut Curie, Paris, which is a member of the French National Research Infrastructure France-BioImaging (ANR-10-INSB-04). The authors thank Hélène Montes for the rheometric measurements of cross-linked PDMS.

\section{References}

[1] Pritchard, R. H., Y. Y. S. Huang, and E. M. Terentjev, "Mechanics of biological networks: From the cell cytoskeleton to connective tissue," Soft Matter 10, 1864-1884 (2014).

[2] Broedersz, C. P., and F. C. MacKintosh, "Modeling semiflexible polymer networks," Rev. Mod. Phys. 86, 995-1036 (2014).

[3] Storm, C., J. J. Pastore, F. C. MacKintosh, T. C. Lubensky, and P. A. Janmey, "Nonlinear elasticity in biological gels," Nature 435, 191-194 (2005)

[4] Licup, A. J., S. Muenster, A. Sharma, M. Sheinman, L. M. Jawerth, B. Fabry, D. A. Weitz, and F. C. MacKintosh, "Stress controls the mechanics of collagen networks," Proc. Natl. Acad. Sci. U. S. A. 112, 9573-9578 (2015).

[5] Williams, B. R., R. A. Gelman, D. C. Poppke, and K. A. Piez, "Collagen fibril formation-optimal in vitro conditions and preliminary kinetic results," J. Biol. Chem. 253, 6578-6585 (1978).

[6] Collagen Structure and Mechanics, edited by P. Fratzl (Springer, New York, 2008).

[7] Streeter, I., and N. H. de Leeuw, "A molecular dynamics study of the interprotein interactions in collagen fibrils," Soft Matter 7, 3373-3382 (2011).

[8] Kadler, K. E., D. F. Holmes, J. A. Trotter, and J. A. Chapman, "Collagen fibril formation," Biochem. J. 316, 1-11 (1996).

[9] Verhulsel, M., M. Vignes, S. Descroix, L. Malaquin, D. M. Vignjevic, and J.-L. Viovy, "A review of microfabrication and hydrogel engineering for micro-organs on chips," Biomaterials 35, 1816-1832 (2014).

[10] Nam, S., K. H. Hu, M. J. Butte, and O. Chaudhuri, "Strain-enhanced stress relaxation impacts nonlinear elasticity in collagen gels," Proc. Natl. Acad. Sci. U.S.A. 113, 5492-5497 (2016).

[11] Raub, C. B., J. Unruh, V. Suresh, T. Krasieva, T. Lindmo, E. Gratton, B. J. Tromberg, and S. C. George, "Image correlation spectroscopy of multiphoton images correlates with collagen mechanical properties," Biophys. J. 94, 2361-2373 (2008).

[12] Yang, Y.-1., L. M. Leone, and L. J. Kaufman, "Elastic moduli of collagen gels can be predicted from two-dimensional confocal microscopy,” Biophys. J. 97, 2051-2060 (2009).

[13] Velegol, D., and F. Lanni, "Cell traction forces on soft biomaterials. I. Microrheology of Type I collagen gels,” Biophys. J. 81, 1786-1792 (2001).

[14] Latinovic, O., L. A. Hough, and H. D. Ou-Yang, "Structural and micromechanical characterization of type I collagen gels," J. Biomech. 43, 500-505 (2010).

[15] Gambini, C., B. Abou, A. Ponton, and A. J. M. Cornelissen, "Microand macrorheology of jellyfish extracellular matrix," Biophys. J. 102, $1-9$ (2012).

[16] Shayegan, M., and N. R. Forde, "Microrheological characterization of collagen systems: from molecular solutions to fibrillar gels," Plos One 8, e70590 (2013).

[17] Wong, L. H., N. A. Kurniawan, H.-P. Too, and R. Rajagopalan, "Spatially resolved microrheology of heterogeneous biopolymer hydrogels using covalently bound microspheres," Biomech. Model. Mechanobiol. 13, 839-849 (2014).

[18] MacKintosh, F. C., and C. F. Schmidt, "Microrheology," Curr. Opin. Colloid Interface Sci. 4, 300-307 (1999).

[19] Bush, B. G., J. M. Shapiro, F. W. DelRio, R. F. Cook, and M. L. Oyen, "Mechanical measurements of heterogeneity and length scale effects in PEG-based hydrogels," Soft Matter 11, 7191-7200 (2015).

[20] Pottier, B., G. Ducouret, C. Fretigny, F. Lequeux, and L. Talini, "High bandwidth linear viscoelastic properties of complex fluids from the measurement of their free surface fluctuations," Soft Matter 7, 7843-7850 (2011). 
[21] Cross, V. L., Y. Zheng, N. W. Choi, S. S. Verbridge, B. A. Sutermaster, L. J. Bonassar, C. Fischbach, and A. D. Stroock, "Dense type I collagen matrices that support cellular remodeling and microfabrication for studies of tumor angiogenesis and vasculogenesis in vitro," Biomaterials 31, 8596-8607 (2010).

[22] Geraldo, S., A. Simon, N. Elkhatib, D. Louvard, L. Fetler, and D. M. Vignjevic, "Do cancer cells have distinct adhesions in 3D collagen matrices and in vivo?," Eur. J. Cell Biol. 91, 930-937 (2012).

[23] Geraldo, S., A. Simon, and D. M. Vignjevic, "Revealing the cytoskeletal organization of invasive cancer cells in 3D," J. Visualized Exp. 80, e50763 (2013).

[24] Artym, V. V., S. Swatkoski, K. Matsumoto, C. B. Campbell, R. J. Petrie, E. K. Dimitriadis, X. Li, S. C. Mueller, T. H. Bugge, M. Gucek, and K. M. Yamada, "Dense fibrillar collagen is a potent inducer of invadopodia via a specific signaling network," J. Cell Biol. 208, 331-350 (2015).

[25] Hackelbusch, S., T. Rossow, P. van Assenbergh, and S. Seiffert, "Chain dynamics in supramolecular polymer networks," Macromolecules 46, 6273-6286 (2013).

[26] Rossow, T., S. Hackelbusch, P. van Assenbergh, and S. Seiffert, "A modular construction kit for supramolecular polymer gels," Polym. Chem. 4, 2515-2527 (2013).

[27] Muller, K. W., R. F. Bruinsma, O. Lieleg, A. R. Bausch, W. A. Wall, and A. J. Levine, "Rheology of semiflexible bundle networks with transient linkers," Phys. Rev. Lett. 112, 238102 (2014).

[28] Tay, A., C. Thibierge, D. Fournier, C. Fretigny, F. Lequeux, C. Monteux, J. P. Roger, and L. Talini, "Probing thermal waves on the free surface of various media: Surface fluctuation specular reflection spectroscopy," Rev. Sci. Instrum. 79, 103107 (2008).
[29] Pottier, B., A. Raudsepp, C. Fretigny, F. Lequeux, J.-F. Palierne, and L. Talini, "High frequency linear rheology of complex fluids measured from their surface thermal fluctuations," J. Rheol. 57, 441-455 (2013).

[30] Crocker, J. C., M. T. Valentine, E. R. Weeks, T. Gisler, P. D. Kaplan, A. G. Yodh, and D. A. Weitz, "Two-point microrheology of inhomogeneous soft materials,” Phys. Rev. Lett. 85, 888-891 (2000).

[31] Jorgensen, L., M. Le Merrer, H. Delanoe-Ayari, and C. Barentin, "Yield stress and elasticity influence on surface tension measurements," Soft Matter 11, 5111-5121 (2015).

[32] Lequeux, F., L. Talini, E. Verneuil, G. Delannoy, and P. Valois, "Wetting of polymers by their solvents," Eur. Phys. J. E 39, 12 (2016).

[33] Deng, L., X. Trepat, J. P. Butler, E. Millet, K. G. Morgan, D. A. Weitz, and J. J. Fredberg, "Fast and slow dynamics of the cytoskeleton," Nat. Mater. 5, 636-640 (2006).

[34] Gittes, F., and F. C. MacKintosh, "Dynamic shear modulus of a semiflexible polymer network," Phys. Rev. E 58, R1241-R1244 (1998).

[35] Morse, D. C., "Viscoelasticity of concentrated isotropic solutions of semiflexible polymers. 2. Linear response," Macromolecules 31, 7044-7067 (1998).

[36] Koenderink, G. H., M. Atakhorrami, F. C. MacKintosh, and C. F. Schmidt, "High-frequency stress relaxation in semiflexible polymer solutions and networks," Phys. Rev. Lett. 96, 138307 (2006).

[37] Cyron, C. J., K. W. Mueller, K. M. Schmoller, A. R. Bausch, W. A. Wall, and R. F. Bruinsma, "Equilibrium phase diagram of semiflexible polymer networks with linkers," EPL 102, 38003 (2013).

[38] Verhulsel, M., "In vitro reproduction of a gut on a microfluidic chip," Ph.D thesis, Université Pierre et Marie Curie, 2015. 